\title{
Hematopoietic stem cell transplantation for thalassemia major using HLA fully-matched and mismatched donor grafts
}

\author{
Chuwen Huang, Yuhua Qu, Sha Liu, Shushan Nie, Hua Jiang \\ Hematology and Oncology Department, Guangzhou Women and Children Medical Center, Guangzhou, China \\ Contributions: (I) Conception and design: C Huang; (II) Administrative support: H Jiang; (III) Provision of study materials or patients: Y Qu; \\ (IV) Collection and assembly of data: S Liu; (V) Data analysis and interpretation: S Nie; (VI) Manuscript writing: All authors; (VII) Final approval of \\ manuscript: All authors. \\ Correspondence to: Hua Jiang. Hematology and Oncology Department, Guangzhou Women and Children Medical Center, Guangzhou, China. \\ Email: jiang_hua18@sina.cn.
}

Background: Until now, allogeneic hematopoietic stem cell transplantation (allo-HSCT) is the only
effective method to cure Thalassemia major. However, it has not been determined whether similar results
can be obtained with the same conditioning regimen for both fully-matched and mismatched donors grafts.
We hypothesized that using modified NF-08-TM conditioning regimen could achieve similar results for
both fully and mismatched donors grafts.
Methods: This retrospective cohort study included patients with $\beta$-thalassemia major who underwent
HSCT with modified NF-08-TM conditioning regimen at Guangzhou Women and Children's Medical
Centre between January 2013 and January 2019 .
Results: Among the 257 patients (172 males) included in this study, 3 had two transplantations. Totally
193 and 67 had fully-matched and mismatched donors were examined, respectively. The median follow-up
was 29 months; 6-year overall survival (OS), thalassemia-free survival (TFS), graft rejection (GR) and
transplantation-related mortality (TRM) were $92.08 \%, 90.89 \%, 1.24 \%$ and $8.01 \%$, respectively. Multivariate
analysis showed that human leukocyte antigen (HLA) compatibility between patient and donor was
not independently associated with OS, TFS, GR or TRM. Mismatched donor graft transplantation for
$\beta$-thalassemia major is associated with similar survival outcomes and incidences of complications (except for
acute GVHD) to fully-matched donor graft transplantation based on modified NF-08-TM conditioning
regimen.

Conclusions: In conclusion, based on the modified NF-08-TM regimen, certain mismatched donor transplantations for thalassemia major patients could achieve similar results as fully-matched donor transplantations.

Keywords: Thalassemia; hematopoietic stem cell transplantation; HLA antigens; transplant donors

Submitted Dec 02, 2020. Accepted for publication Apr 21, 2021.

doi: $10.21037 / \mathrm{tp}-20-415$

View this article at: http://dx.doi.org/10.21037/tp-20-415

\section{Introduction}

$\beta$-thalassemia is a genetic disorder characterized by impaired or absent synthesis of the $\beta$-globin chains of hemoglobin (1). $\beta$-thalassemia major is associated with the most severe clinical manifestations, including severe anemia and hepatosplenomegaly. Current clinical management of $\beta$-thalassemia major includes regular transfusion and iron chelation (2). Although research into gene therapy is ongoing, the only current curative treatment for $\beta$-thalassemia is allo-HSCT $(2,3)$.

The majority of patients treated with allo-HSCT show a good response to treatment, and recent advancements in transplantation technology have improved the OS and 
TFS rates to around $90 \%$ and $80 \%$, respectively $(4,5)$. Most transplants use HLA fully-matched sibling donors, but limitations in the number of matched related donors has led to the use of mismatched related donors, matched unrelated donors and mismatched unrelated donors (6). Although research has indicated that good results can be achieved with mismatched donors, the outcomes tend to be worse than those obtained with matched donors for both $\beta$-thalassemia (7-9) and other diseases $(10,11)$. Besides, based on the same conditioning regimen, whether the HSCT of mismatched donors can achieve similar effects as that of fully-matched donors for $\beta$-thalassemia major remains unclear.

A recent study of 82 patients with $\beta$-thalassemia in China reported that 3 -year OS and TFS rates of up to $90 \%$ and $85 \%$, respectively, could be achieved using the novel NF08-TM conditioning regimen (12). However, the above study included $73.2 \%$ fully-matched donors, $25.6 \% 7 / 8$ matched donors, $1.2 \%$ 6/8 matched donors and patients considered to be at low-to-middle risk (12). Furthermore, thiotepa is not approved for application in our hospital because of a policy prohibiting the use of self-purchased drugs by patients, necessitating further clinical research into alternative conditioning regimens. Meanwhile, considering that the major causes of transplantation failure of HLA mismatched donors include severe GVHD and treatmentrelated death, we speculated a regimen adjusting the ATG dose based on different HLA allele mismatched and a total cyclophosphamide dose of $120 \mathrm{mg} / \mathrm{kg}$ could reduce the rate of GVHD and treatment related death, facilitating engraftment. Therefore, we hypothesized that a modified NF-08-TM regimen could achieve similar outcomes as the NF-08-TM regimen in patients with $\beta$-thalassemia undergoing HSCT. The aim of this study was to evaluate survival outcomes and complications in patients with $\beta$-thalassemia administered HSCT with matched or mismatched donor grafts using the modified NF-08-TM protocol.

We present the following article in accordance with the STROBE reporting checklist (available at http://dx.doi. org/10.21037/tp-20-415).

\section{Methods}

\section{Patients}

This retrospective cohort study included patients with $\beta$-thalassemia major who underwent allo-HSCT between January 2013 and January 2019 at the Department of
Hematologic Oncology, Guangzhou Women and Children's Medical Centre. Inclusion criteria were: (I) age $<18$ years; (II) routine hematology demonstrating severe anemia; (III) $\beta$-thalassemia major diagnosed based on blood transfusiondependence before the age of 2 years, hemoglobin levels $<70 \mathrm{~g} / \mathrm{L}$ before transfusion and genetic testing (13); (IV) peripheral blood stem transplantation using the modified NF-08-TM regimen; (V) HLA fully-matched or 1 to 2 locus-mismatched donor (14), 3 or more locus mismatched relative donor. Exclusion criteria were: (I) hepatitis; (II) abnormal liver or renal function before HSCT; (III) severe bacterial, fungal or viral infection before HSCT. The study was conducted in accordance with the Declaration of Helsinki (as revised in 2013). It was approved by the ethics committee of Guangzhou Women and Children's Medical Center (No. 2013092569), and individual consent for this retrospective analysis was waived.

\section{Determination of risk class}

Pesaro risk class is difficult to apply in China because most patients refuse liver biopsy owing to Chinese traditional concepts. NF-08-TM risk class was defined according to the criteria described by Li et al. (12), which are suitable for Chinese individuals. Class I included patients who were $<4$ years old with a liver edge extending $<2.5 \mathrm{~cm}$ below the costal margin and serum ferritin levels $<3,000 \mathrm{mg} / \mathrm{dL}$. Class III included patients who were $>8$ years old with a liver edge extending $>4 \mathrm{~cm}$ below the costal margin and serum ferritin levels $>5,000 \mathrm{mg} / \mathrm{dL}$. Patients who did not meet criteria for class I or II were categorized as class III.

\section{Stem cell collection and processing}

Peripheral blood stem cell mobilization was performed by administration of granulocyte colony stimulating factors (G-CSF) for 4 days at $10 \mathrm{mcg} / \mathrm{kg} / \mathrm{day}$, and the collection occurred on the 5 th day with a peripheral blood stem cell apheresis unit. Blood counts were obtained daily to assess total peripheral blood WBC count. Peripheral blood smear and CD34 level assessment were performed prior to the harvest. Either of the central veins were cannulated for vascular access with a single lumen catheter of appropriate size, according to patient age, and used as the access line. Stem cell harvest was performed with a continuous flow blood cell separator $\left(\mathrm{COBE}^{\circledR}\right.$ Spectra $\left.{ }^{\mathrm{TM}} \mathrm{PBSC}^{\mathrm{TM}}\right)$. The PSBC apheresis kit $\left(\mathrm{COBE}^{\circledR}\right.$ Spectra Apheresis) was loaded onto the apheresis machine. The weight, height, hematocrit 
Table 1 Precondition regimens

\begin{tabular}{|c|c|c|c|c|c|c|c|c|c|c|c|c|c|}
\hline Regimen & Day & \multicolumn{6}{|c|}{ NF-08-TM } & Day & \multicolumn{5}{|c|}{ Modified NF-08-TM } \\
\hline Cy (mg/kg/d) & -10 to -9 & \multicolumn{2}{|c|}{60} & \multicolumn{2}{|c|}{55} & \multicolumn{2}{|c|}{50} & -10 to -9 & & & 60 & & \\
\hline \multirow[t]{2}{*}{$\mathrm{Bu}(\mathrm{mg} / \mathrm{kg} / \mathrm{d})$} & -8 to -6 & $1-2 y$ & 4.4 & $<6 y$ & 3.6 & $<8 \mathrm{y}$ & 3 & -8 to -5 & $<9 \mathrm{~kg}$ & $9-16 \mathrm{~kg}$ & $16-23 \mathrm{~kg}$ & $23-34 \mathrm{~kg}$ & $>34 \mathrm{~kg}$ \\
\hline & & $>2 y$ & 4 & $>6 \mathrm{y}$ & 3.2 & $>8 \mathrm{y}$ & 3.2 & & 4 & 4.8 & 4.4 & 3.8 & 3.2 \\
\hline Flu $\left(\mathrm{mg} / \mathrm{m}^{2} / \mathrm{d}\right)$ & -8 to -4 & \multicolumn{6}{|c|}{40} & -8 to -4 & \multicolumn{5}{|c|}{40} \\
\hline \multirow[t]{2}{*}{ Total ATG (mg/kg) } & -3 to -2 & & & 7.5 & & & & -3 to -2 & MFD & MUD & 1-2 HLA & 3-4 HLA & \\
\hline & & & & & & & & & & \multicolumn{4}{|c|}{$\begin{array}{l}\text { Allele-mismatched } \\
\text { donor }\end{array}$} \\
\hline
\end{tabular}

and gender of the donor were entered into the PBSC system. The collection rate calculated by the machine and hematopoietic progenitor cells were collected in the collection bag. Total cell count in the harvested product was estimated on an automated cell counter. Manually, MNC\% was estimated (proportion of uni-nucleated cells including lymphocytes and monocytes) and used to derive MNC cell dose. Donors were given oral calcium as prophylaxis prior to the harvest procedure.

\section{Conditioning regimen}

Since thiotepa is unavailable in our hospital, the original NF-08-TM myeloablative conditioning regimen (12) was modified to replace thiotepa on day -5 (i.e., 5 days before HSCT) with busulfan. The modified NF-08-TM regimen consisted of cyclophosphamide at $60 \mathrm{mg} / \mathrm{kg} /$ day on days -10 and -9 ; fludarabine at $40 \mathrm{mg} / \mathrm{m}^{2} /$ day on days -8 to -4 ; busulfan on days -8 to $-5(3.2 \mathrm{mg} / \mathrm{kg} / \mathrm{d}$ for patients weighing $>34 \mathrm{~kg}, 3.8 \mathrm{mg} / \mathrm{kg} / \mathrm{d}$ for those weighing $23-34 \mathrm{~kg}$, $4.4 \mathrm{mg} / \mathrm{kg} / \mathrm{d}$ for those weighing $16-23 \mathrm{~kg}, 4.8 \mathrm{mg} / \mathrm{kg} / \mathrm{d}$ for those weighing $9-16 \mathrm{~kg}$, and $4.0 \mathrm{mg} / \mathrm{kg} / \mathrm{d}$ for those weighing <9 kg) (15-17); and rabbit anti-human thymocyte immunoglobulin (ATG, Genzyme Polyclonals SAS) days -4 and -2 (total dosages of $7.5 \mathrm{mg} / \mathrm{kg}$ for matched family donor grafts, $8.5 \mathrm{mg} / \mathrm{kg}$ for matched unrelated donor grafts, $9 \mathrm{mg} / \mathrm{kg}$ for one or two HLA allele-mismatched grafts, and $10 \mathrm{mg} / \mathrm{kg}$ for three or four HLA allele-mismatched grafts) (18-20). All patients received azathioprine at $3 \mathrm{mg} / \mathrm{kg} / \mathrm{d}$ and hydroxyurea at $30 \mathrm{mg} / \mathrm{kg} / \mathrm{d}$ on days -45 to -11 , as shown in
Table 1.

\section{Prophylaxis for GVHD}

GVHD prophylaxis consisted of cyclosporine A (CSA) mycophenolate mofetil (MMF) and methotrexate (MTX) in all patients (12). CSA was intravenously injected from -10 to -2 days at a dose of $1.5 \mathrm{mg} / \mathrm{kg}$.d., and changed to $3 \mathrm{mg} / \mathrm{kg}$.d from -1 to +25 days. Finally, it was orally administered to maintain the blood concentration at $200 \pm 50 \mathrm{ng} / \mathrm{mL}$. The dose of CSA was tapered from day 180. MMF was administered sequentially from day +1 to day +30 at a dose of $15 \mathrm{mg} / \mathrm{kg}$.d in case of no more than grade 2 acute GVHD. Short-term MTX was administered intravenously on $+1,+3$, and +6 days after transplantation, at $15 \mathrm{mg} / \mathrm{m}^{2}$, $10 \mathrm{mg} / \mathrm{m}^{2}$, and $10 \mathrm{mg} / \mathrm{m}^{2}$ respectively.

\section{Supportive care and prophylaxis against complications}

Alprostadil and limitation of fluid intake $(50 \mathrm{~mL} / \mathrm{kg} . \mathrm{d})$ were used for the prophylaxis of hepatic veno-occlusive disease (VOD). Immunoglobulin was administered at $500 \mathrm{mg} / \mathrm{kg}$ weekly until myeloid engraftment after the transplantation. Ganciclovir at a dose of $10 \mathrm{mg} / \mathrm{kg}$.d was given when the conditioning regimen was started until 2 days before stem cell transfusion, and then changed to acyclovir at a dose of $30 \mathrm{mg} / \mathrm{kg}$.d as prophylaxis against viruses until 3 months after transplantation. No standard prophylactic broadspectrum bacterial antibiotic was administered to patients with neutropenia. The prophylaxis of fungal infections 
with orally administered fluconazole at a dose of $5 \mathrm{mg} / \mathrm{kg}$.d started when the conditioning regimen was started, and continued until engraftment. The thresholds of RBC and platelet transfusions were hemoglobin $<10 \mathrm{~g} / \mathrm{dL}$ and platelets $<20 \times 10^{9} / \mathrm{L}$, respectively. All blood products except the allograft were filtered before transfusion.

\section{Follow-up and endpoints}

The follow-up period ended in February 2020. The median follow-up was 29 months. Donor chimerism levels were analyzed by a polymerase chain reaction-based analysis of short tandem repeats in donors of identical sex or fluorescence in situ hybridization of a $\mathrm{Y}$ chromosome marker in sex-mismatched donors. Complete chimerism was defined as the detection of more than $95 \%$ donorderived cells in peripheral blood samples. Mixed chimerism was defined as a level of donor-derived cells between $10 \%$ and $95 \%$, which was confirmed by testing peripheral blood samples simultaneously or performing at least two consecutive analyses within one week. Graft rejection was defined as $<10 \%$ of donor-derived cells. Chimerism monitoring was initiated upon confirmation of neutrophil and platelet engraftment. Neutrophil engraftment was defined as a neutrophil count $>0.5 \times 10^{9} / \mathrm{L}$ for three consecutive days; platelet engraftment was defined as a platelet count $>20 \times 10^{9} / \mathrm{L}$ at least 7 days after the most recent platelet transfusion. Acute GVHD (aGVHD) was defined as GVHD occurring within 100 days after transplantation, and the classification referred to the 1994 diagnosis consensus (divided into grades I to IV) (21). Chronic GVHD (cGVHD) was defined as GVHD occurring more than 100 days after transplantation, mainly based on the 2003 revised version of Seattle's diagnosis and classification criteria (22). OS was defined as the time from HSCT to death or the last follow-up. TFS was defined as the time from HSCT to recurrent transfusion-dependent thalassemia, death or the last follow-up. GR was defined as recurrent thalassemia and chimerism studies showing no donor cells or a return to a blood transfusion-dependent status after transplantation. TRM was defined as death related to transplantation but not to recurrence of thalassemia.

\section{Statistical analysis}

SPSS 20.0 (IBM Corp, Armonk, NY, USA) was used for data analysis. Categorical variables were presented as count and percentage. Continuous variables were presented as median and range. Continuous variables were compared between groups by the $t$-test or the Mann-Whitney $\mathrm{U}$ test. Categorical variables were compared between groups by the Pearson's chi-squared or Fisher's exact test. OS, TFS, GR and TRM were determined by the Kaplan-Meier productlimit method, and the impact of a given variable was assessed by the log-rank test. A multivariable Cox proportional hazards regression model was used to determine the effects of factors that were statistically significant in univariate analysis, as well as other factors considered clinically important. Multivariate Cox regression analysis determining hazard ratios (HRs) and 95\% confidence intervals (CIs) was performed to identify factors independently associated with OS, TFS, GR and TRM. Two-sided $\mathrm{P}<0.05$ was considered statistically significant.

\section{Results}

\section{Clinical characteristics of the study participants}

The study included a total of 257 patients (172 males, $66.9 \%)$ with median age of $6[4-8]$ years. Totally 3 patients had two transplantations for graft failure. No patient was lost to follow-up. Of all 260 transplantations, NF-08-TM risk class was I in 23 patients (8.8\%), II in 212 (81.5\%) and III in 25 (9.6\%). Before transplantation, the lower border of the spleen was palpable $<1 \mathrm{~cm}$ below the costal margin in 85 cases (32.7\%), $1-3 \mathrm{~cm}$ in $77(29.6 \%)$ and $>3 \mathrm{~cm}$ in 78 $(30.0 \%) ; 20$ cases $(7.7 \%)$ had undergone prior splenectomy. Among the 260 cases with available data, left ventricular ejection fraction (LVEF) before transplantation was $\leq 60 \%$ in 28 cases $(10.8 \%)$ and $>60 \%$ in $232(89.2 \%)$.

\section{Donor and stem cell source}

There were 145 male and 115 female donors. Among the 260 study transplantations, 193 patients had fully-matched donors (including 105 family and 88 unrelated donors) and 67 had mismatched donors (including 28 family and 39 unrelated donors).

Among the 193 patients with fully-matched donors, only 1 had 6/6 sibling donor with allelic matched occurring at the HLA-A, HLA-B and HLA-DRB1 locus owing to the detection technology at the time; 10 patients had $8 / 8$ matched donors from Tzu Chi's Taiwan bone marrow bank with allelic matched occurring at the HLA-A, HLA-B, HLA-C and HLA-DRB1 locus. The remaining patients had 10/10 matched donors with allelic matched occurring at the 
Table 2 Baseline clinical characteristics of the 260 study transplantations

\begin{tabular}{|c|c|c|c|}
\hline Characteristic & Fully-matched donor group $(n=193)$ & Mismatched donor group $(n=67)$ & $\mathrm{P}$ \\
\hline Male $(n, \%)$ & $130(67.36 \%)$ & $34(64.18 \%)$ & 0.635 \\
\hline Ferritin level (ng/mL) & $3,412(2,306-4,845)$ & $3,246(2,348-4,606)$ & 0.630 \\
\hline Liver span below right costal margin $(\mathrm{cm})$ & $3(2.0-3.5)$ & $3(2.5-4)$ & 0.594 \\
\hline Spleen span below left costal margin $(\mathrm{cm})$ & $2(1.5-3)$ & $2(1-3.5)$ & 0.289 \\
\hline $\begin{array}{l}\text { Mononuclear cells infused during transplantation } \\
\left(\times 10^{8} / \mathrm{kg} \text { body weight of the recipient) }\right.\end{array}$ & $8.06(8.00-8.49)$ & $8.00(8.00-8.42)$ & 0.101 \\
\hline $\begin{array}{l}\text { CD34 cells infused during transplantation } \\
\left(\times 10^{6} / \mathrm{kg} \text { body weight of the recipient }\right)\end{array}$ & $5.76(4.07-7.64)$ & $6.36(4.73-9.00)$ & 0.072 \\
\hline Class I & $17(8.7 \%)$ & $6(9.2 \%)$ & \\
\hline Class II & $155(81.0 \%)$ & $57(84.6 \%)$ & \\
\hline Class III & $21(10.3 \%)$ & $4(6.2 \%)$ & \\
\hline
\end{tabular}

HLA-A, HLA-B, HLA-C, HLA-DRB1 and HLA-DQB1 locus.

Among the 67 patients with mismatched donors, 45 received one HLA-allele mismatched graft (allelic mismatches occurred at the HLA-A locus in 17 donorrecipient pairs, at the HLA-B locus in 2, at the HLA-C locus in 7, at the HLA-DRB1 locus in 11, and at the HLADQB1 locus in 8). Totally 22 patients received two or more HLA-allele mismatched grafts, including 19 patients who received two HLA-allele mismatched grafts (allelic mismatches occurred at the HLA-DRB1 and HLA-DQB1 locus in 12 donor-recipient pairs, at the HLA-A and HLA-DQB1 locus in 2, at the HLA-C and HLA-DQB1 locus in 2, at the HLA-A and HLA-C locus in 1, at the HLA-A and HLA-DRB1 locus in 1, and at the HLA-B and HLA-C locus in 1), 2 patients who received three HLAallele mismatched grafts (allelic mismatches occurred at the HLA-A, HLA-DRB1 and HLA-DQB1 locus in 1 donor-recipient pair from the father and at the HLA-A, HLA-B and HLA-C locus in 1 donor-recipient pair from the mother) and 1 patient who received four HLA-allele mismatched graft with HLA-B, HLA-C, HLA-DRB1 and HLA-DQB1 locus mismatched from the father.

There were no significant differences in age, sex, ferritin levels, liver size, spleen size, LVEF, amounts of mononuclear cells or CD34 cells, infusion, relationship, class risk between patients with fully-matched donors and those with mismatched donors (Table 2).

\section{Engraftment}

Three patients underwent twice transplantations owing to graft failure and achieved both neutrophil and platelet engraftment at the second transplantation; the remaining patients successfully achieved both neutrophil and platelet engraftment. The median times to neutrophil and platelet engraftment were 11 [11-12] days and 12 [10-13] days, respectively. There were no significant differences between the fully-matched and mismatched donor groups in time to neutrophil or platelet engraftment (Table 3). Notably, subgroup analysis of patients in the mismatched donor group demonstrated that times to neutrophil and platelet engraftment were not significantly different in individuals with one and $>1$ HLA-allele mismatched donors (Table 4).

\section{Survival}

Among the 257 study participants, 237 (92.2\%) were still alive at the end of the follow-up, and $236(91.8 \%)$ were thalassemia-free. Three patients $(1.2 \%)$ developed 
Table 3 Complications in the fully-matched and mismatched donor groups.

\begin{tabular}{lcc}
\hline Endpoint & Fully-matched donor group $(\mathrm{n}=193)$ & Mismatched donor group $(\mathrm{n}=67)$ \\
\hline Time to neutrophil engraftment (days) & $11(11.00-12.00)$ & $12(11.00-12.00)$ \\
Time to platelet engraftment (days) & $12(10.00-13.00)$ & $12(11.00-12.00)$ \\
Complications, $n(\%)$ & & $33(49.25 \%)$ \\
aGVHD & $48(24.87 \%)$ & $10(14.93 \%)$ \\
cGVHD & $17(8.81 \%)$ & $8(11.94 \%)$ \\
PRES & $13(6.74 \%)$ & $20(29.85 \%)$ \\
HC & $46(23.83 \%)$ & $17(25.37 \%)$ \\
AlHA & $37(19.17 \%)$ & $6(8.96 \%)$ \\
BO & $8(4.15 \%)$ & 0.157 \\
PTLD & $1(0.52 \%)$ & 0.17802 \\
CMV infection & $50(25.91 \%)$ & 0.330 \\
EBV infection & $30(15.54 \%)$ & $21(31.34 \%)$ \\
VOD & $4(2.07 \%)$ & $15(22.39 \%)$ \\
\hline
\end{tabular}

GVHD, graft versus host disease; PRES, posterior reversible encephalopathy syndrome; HC, hemorrhagic cystitis; AlHA, autoimmune hemolytic anemia; BO, bronchiolitis obliterans; PTLD, posttransplant lymphoproliferative disorders; EBV, Epstein-Barr virus; VOD, vein occlusive disease.

Table 4 Complications in patients with different HLA-allele mismatched grafts

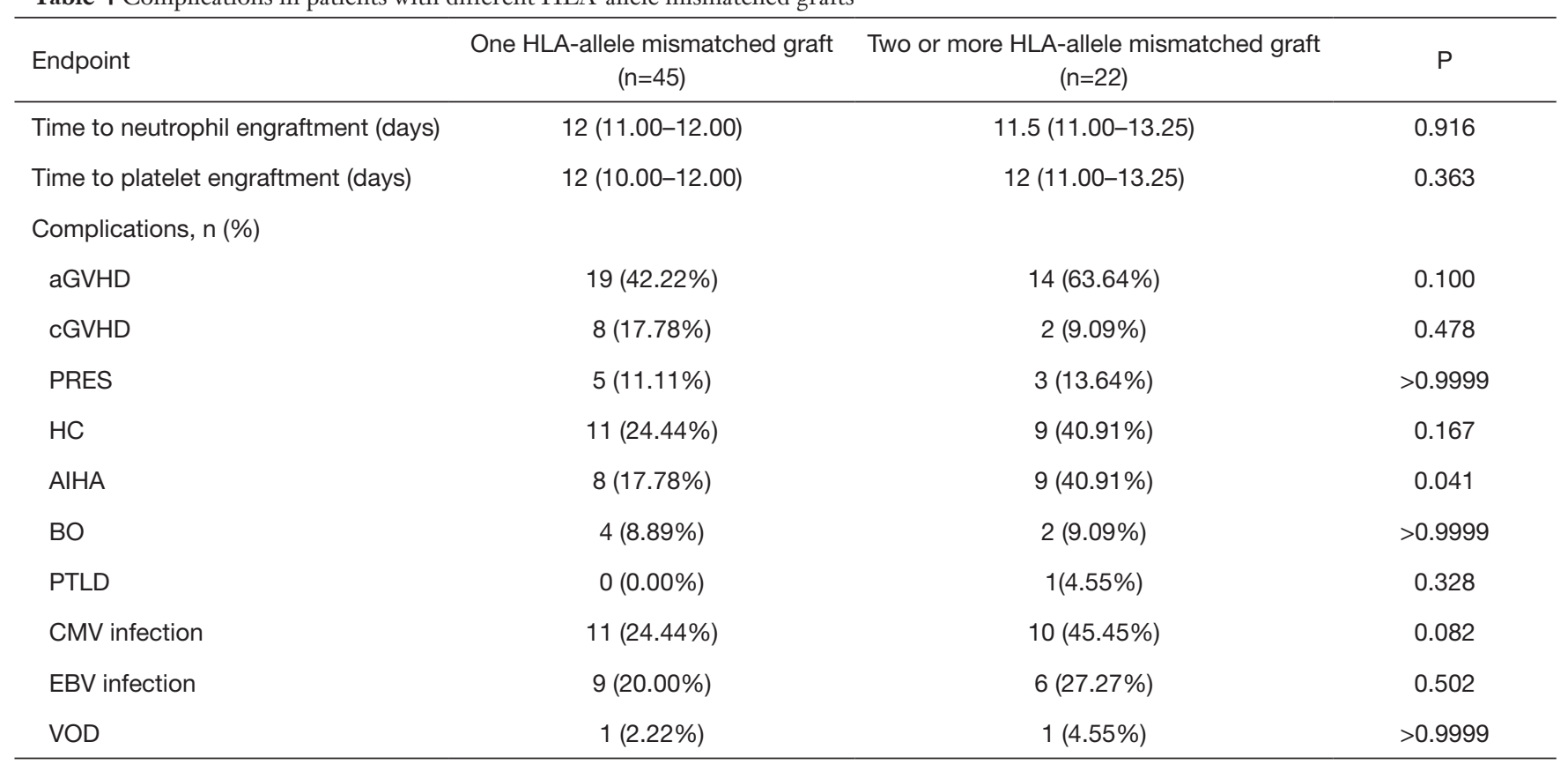

GVHD, graft versus host disease; PRES, posterior reversible encephalopathy syndrome; HC, hemorrhagic cystitis; AlHA, autoimmune hemolytic anemia; BO, bronchiolitis obliterans; PTLD, posttransplant lymphoproliferative disorders; EBV, Epstein-Barr virus; VOD, vein occlusive disease. 
Overall OS, TFS, TRM, GR

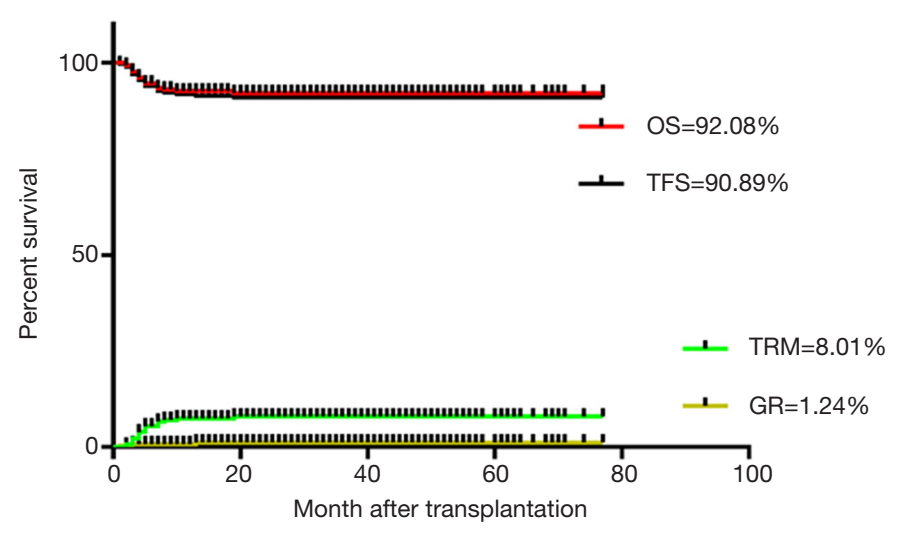

Figure 1 Overall OS, TFS, TRM and GR analyses. OS, overall survival; TFS, thalassemia-free survival; GR, graft rejection; TRM, transplantation-related mortality.

Table 5 Clinical endpoints in the fully-matched and mismatched donor groups.

\begin{tabular}{lcccc}
\hline Endpoint & $\begin{array}{c}\text { Fully-matched donor group } \\
(\mathrm{n}=193)\end{array}$ & $\begin{array}{c}\text { Mismatched donor group } \\
(\mathrm{n}=67)\end{array}$ & $\mathrm{HR}$ & $95 \% \mathrm{Cl}$ \\
\hline OS (\%) & $93.15 \%$ & $89.04 \%$ & 0.58 & $0.21-1.60$ \\
TFS (\%) & $91.53 \%$ & $89.04 \%$ & 0.75 & $0.29-1.94$ \\
GR (\%) & $1.66 \%$ & $0.00 \%$ & 3.83 & 0.293 \\
TRM (\%) & $6.96 \%$ & $10.96 \%$ & 0.59 & $0.21-1.63$ \\
\hline
\end{tabular}

OS, overall survival; TFS, thalassemia-free survival; GR, graft rejection; TRM, transplantation-related mortality.

graft rejection at the first transplantation, and all of them underwent a second transplantation based on the same regimen. Meanwhile, they all achieved stable chimerism. A total of 20 patients $(7.8 \%)$ died, including 15 due to severe pneumonia (two of them underwent a second transplantation), 2 due to intracranial hemorrhage secondary to thrombocytopenia, 1 due to pulmonary hemorrhage secondary to thrombocytopenia, 1 due to respiratory failure secondary to myasthenia gravis and 1 due to cardiac arrest following an unintentional self-overdose of nifedipine. The median follow-up was 29 months, and 6-year OS, TFS, GR and TRM were $92.08 \%$, 90.89\%, $1.24 \%$ and $8.01 \%$, respectively, as shown in Figure 1.

Comparisons of OS, TFS, GR and TRM between the fully-matched and mismatched donor groups are shown in Table 5 and Figure 2. Notably, there were no significant differences between the fully-matched and mismatched donor groups in 6-year OS (93.15\% vs. $89.04 \%)$, TFS (91.53\% vs. $89.04 \%)$, GR (1.66\% vs. $0.00 \%)$ and TRM (6.96\% vs. $10.96 \%)$.
As shown in Table 6 and Figure 3, patients administered one and two or more HLA allele-mismatched grafts exhibited no significant differences in 6-year OS $(92.57 \%$ vs. 81.57$)$, TFS (92.57\% vs. 81.57$)$, TRM (7.41\% vs. $18.43 \%)$. Neither of them underwent GR.

There were no significant differences between the fully-matched family and unrelated donor subgroups in 6-year OS (94.24\% vs. 91.83\%, $\mathrm{P}=0.541)$, TFS $(93.19 \%$ vs. $89.55 \%, \mathrm{P}=0.358)$, GR $(1.11 \%$ vs. $2.27 \%, \mathrm{P}=0.446)$ and TRM (5.82\% vs. $8.36 \%, \mathrm{P}=0.523)$. Similarly, there were no significant differences between the mismatched family and unrelated donor subgroups in 6-year OS (85.57\% vs. $91.48 \%, \mathrm{P}=0.367)$, TFS (85.57\% vs. $91.48 \%, \mathrm{P}=0.367)$, GR (0.00\% vs. $0.00 \%, \mathrm{P}>0.9999)$ and TRM (14.44\% vs. $8.52 \%$, $\mathrm{P}=0.367$ ), as shown in Table 7 .

\section{Complication comparisons between the fully-matched and mismatched groups}

Comparisons of aGVHD, cGVHD, posterior reversible 

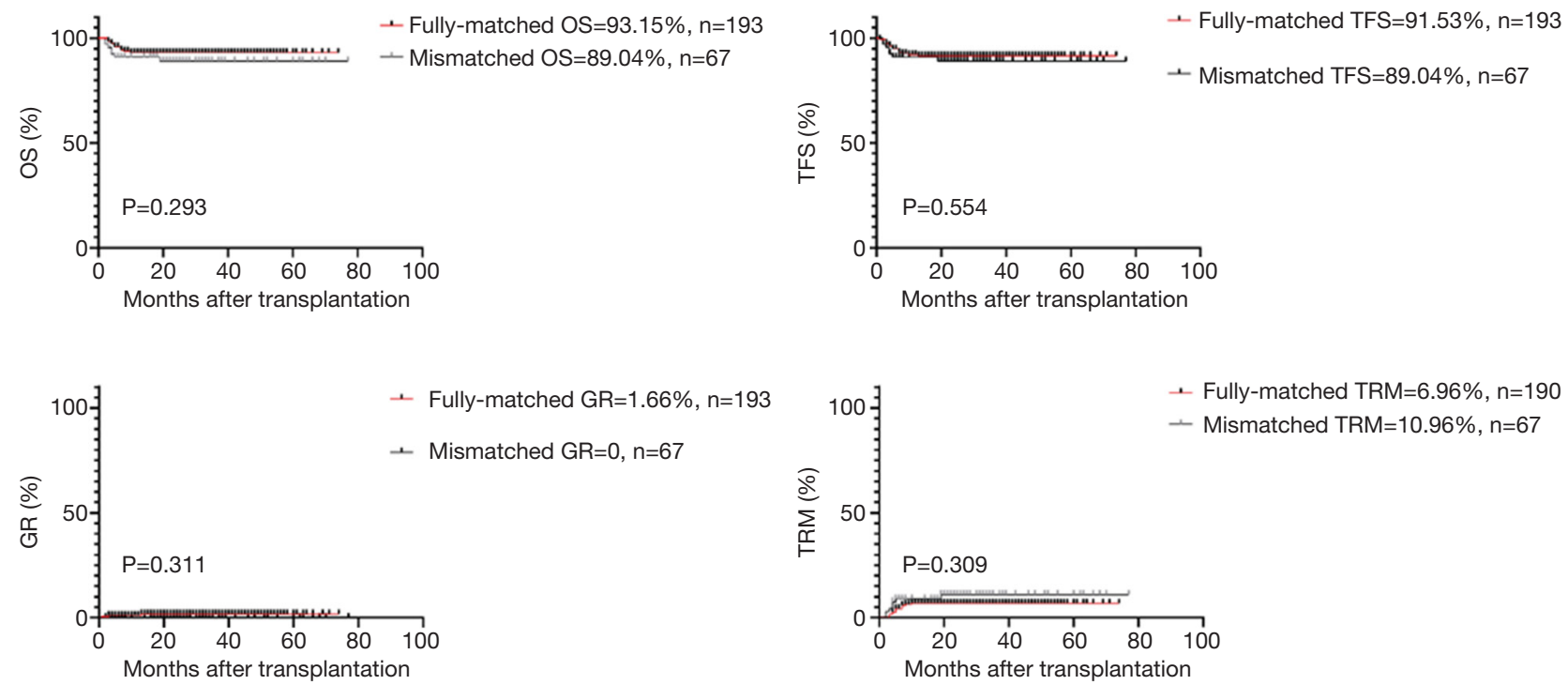

Figure 2 OS, TFS, TRM and GR in the fully-matched and mismatched groups. OS, overall survival; TFS, thalassemia-free survival; GR, graft rejection; TRM, transplantation-related mortality.

Table 6 Clinical endpoints in patients with different HLA-allele mismatched grafts

\begin{tabular}{lcccc}
\hline Endpoint & $\begin{array}{c}\text { One HLA-allele mismatched graft } \\
(\mathrm{n}=45)\end{array}$ & $\begin{array}{c}\text { Two or more HLA-allele mismatched graft } \\
(\mathrm{n}=22)\end{array}$ & $\mathrm{HR}$ & $95 \% \mathrm{Cl}$ \\
\hline OS (\%) & 92.57 & 81.57 & 0.3 & $0.06-1.50$ \\
TFS (\%) & 92.57 & 81.57 & 0.3 & $0.06-1.50$ \\
GR (\%) & 0 & 0 & 0.142 & 0.3 \\
TRM (\%) & 7.41 & 18.43 & $0.06-1.50$ & 0.142 \\
\hline
\end{tabular}

OS, overall survival; TFS, thalassemia-free survival; GR, graft rejection; TRM, transplantation-related mortality.

encephalopathy syndrome (PRES), hemorrhagic cystitis (HC), autoimmune hemolytic anemia (AIHA), bronchiolitis obliterans (BO), posttransplant lymphoproliferative disorders (PTLD), cytomegalovirus infection, Epstein-Barr virus infection and hepatic vein occlusive disease (VOD) between the fully-matched and mismatched donor groups are shown in Table 3. Acute GVHD incidence was higher in the mismatched group compared with the fully-matched group $(49.25 \%$ vs. $24.87 \%, \mathrm{P}=0.0002)$. Notably, there were no significant differences between the fully-matched and mismatched donor groups in the incidence rates of cGVHD (8.81\% vs. $14.93 \%, \mathrm{P}=0.157)$, PRES (6.74\% vs. $11.94 \%$, $\mathrm{P}=0.178)$, HC (23.83\% vs. $29.85 \%, \mathrm{P}=0.330)$, AIHA (19.17\% vs.25.37\%, $\mathrm{P}=0.281)$, $\mathrm{BO}(4.15 \%$ vs. $8.96 \%, \mathrm{P}=0.133)$, PTLD (0.52\% vs. $1.49 \%, \mathrm{P}=0.450)$, CMV infection $(25.91 \%$ vs. $31.34 \%, \mathrm{P}=0.390)$, EBV infection $(15.54 \%$ vs. $22.39 \%$,
$\mathrm{P}=0.202)$ and $\operatorname{VOD}(2.07 \%$ vs. $2.99 \%, \mathrm{P}=0.650)$.

As shown in Table 4, patients who received one and two or more HLA allele-mismatched grafts exhibited no significant differences in the incidence rates of aGVHD (42.22\% vs. 63.64\%), cGVHD (17.78\% vs. 9.09\%), PRES (11.11\% vs. $13.64 \%)$, HC (24.44\% vs. $40.91 \%)$, BO (8.89\% vs. $9.09 \%)$, PTLD (0.00\% vs. $4.55 \%)$, CMV infection (24.44\% vs. $45.45 \%)$, EBV infection (20.00\% vs. $27.27 \%)$ and VOD (2.22\% vs. $4.55 \%)$. AIHA incidence was higher in the two or more HLA allele-mismatched group compared with the one HLA allele-mismatched group (40.91\% vs. $17.78 \%, \mathrm{P}=0.041$ ).

As shown in Table 8, patients administered fully-matched family and fully-matched unrelated donor grafts exhibited no significant differences in the incidence rates of cGVHD, HC, BO, PTLD, AIHA, CMV infection, EBV infection 

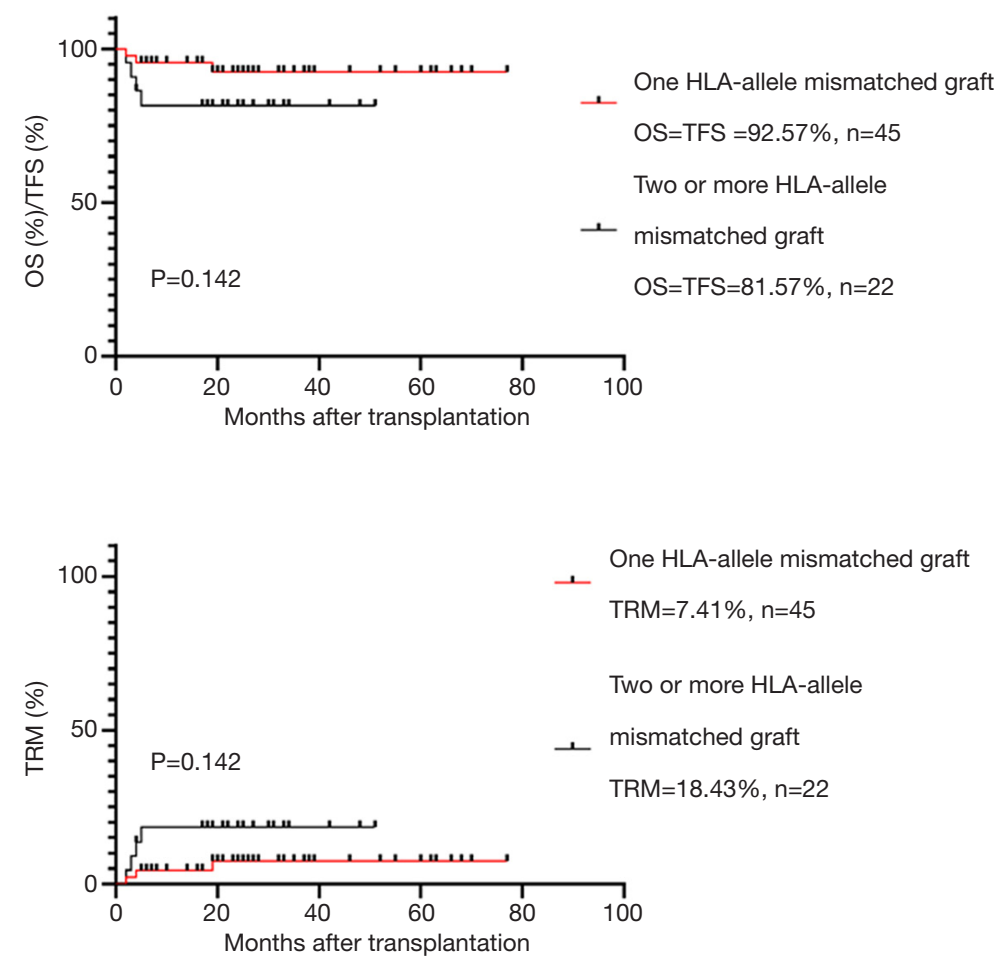

Figure 3 OS and TFS in the one and two or more HLA-allele mismatched donor transplantation groups. OS, overall survival; TFS, thalassemia-free survival.

Table 7 Clinical endpoints in the fully-matched family, fully-matched unrelated, mismatched family, mismatched unrelated donor groups.

\begin{tabular}{|c|c|c|c|c|c|c|c|c|c|c|}
\hline Endpoint & $\begin{array}{l}\text { Fully-matched } \\
\text { family donor } \\
\text { group }(n=105)\end{array}$ & $\begin{array}{l}\text { Fully-matched } \\
\text { unrelated donor } \\
\text { group }(\mathrm{n}=88)\end{array}$ & $\mathrm{HR}$ & $95 \% \mathrm{Cl}$ & $\mathrm{P}$ & $\begin{array}{l}\text { Mismatched } \\
\text { family donor } \\
\text { group }(n=28)\end{array}$ & $\begin{array}{l}\text { Mismatched } \\
\text { unrelated donor } \\
\text { group }(n=39)\end{array}$ & $\mathrm{HR}$ & $95 \% \mathrm{Cl}$ & $P$ \\
\hline TFS (\%) & $93.19 \%$ & $89.55 \%$ & 0.63 & $0.23-1.69$ & 0.358 & $85.56 \%$ & $91.48 \%$ & 2.01 & $0.44-9.16$ & 0.367 \\
\hline GR (\%) & $1.11 \%$ & $2.27 \%$ & 0.41 & $0.04-4.02$ & 0.446 & $0.00 \%$ & $0.00 \%$ & & & $>0.9999$ \\
\hline
\end{tabular}

and VOD. Acute GVHD (35.23\% vs. $16.19 \%, \mathrm{P}=0.002)$ and PRES $(11.36 \%$ vs. $2.86 \%, \mathrm{P}=0.022)$ incidence rates were higher in the fully-matched unrelated donor group compared with the fully-matched family donor group. Patients administered HLA-mismatched family and HLAmismatched unrelated donor grafts exhibited no significant differences in the incidence rates of aGVHD, cGVHD, HC, PTLD, CMV infection, EBV infection and VOD. However, AIHA (39.29\% vs. 15.38\%, $\mathrm{P}=0.027)$ and $\mathrm{BO}$ (39.29\% vs. $7.69 \%, \mathrm{P}=0.002$ ) incidence rates were higher in the HLA-mismatched family donor group compared with the HLA-mismatched unrelated donor group.

\section{Multivariate analysis of factors associated with OS, TFS, GR and TRM}

The factors included in the multivariate analysis were sex, risk class, LVEF, spleen size before HSCT, HLA compatibility, times to neutrophil and platelet engraftment, amounts of mononuclear cells and CD34 cells transplanted, and complications after HSCT. Notably, HLA compatibility was not independently associated with OS, TFS, GR and 
Table 8 Complications in the fully-matched family, fully-matched unrelated, mismatched family and mismatched unrelated donor groups.

\begin{tabular}{|c|c|c|c|c|c|c|}
\hline Endpoint & $\begin{array}{l}\text { Fully-matched family } \\
\text { donor group }(n=105)\end{array}$ & $\begin{array}{l}\text { Fully-matched unrelated } \\
\text { donor group }(n=88)\end{array}$ & $\mathrm{P}$ & $\begin{array}{l}\text { Mismatched family } \\
\text { donor group }(n=28)\end{array}$ & $\begin{array}{l}\text { Mismatched unrelated } \\
\text { donor group }(n=39)\end{array}$ & $\mathrm{P}$ \\
\hline $\begin{array}{l}\text { Time to neutrophil } \\
\text { engraftment (days) }\end{array}$ & $11[11-12]$ & $12[11-14]$ & 0.003 & $11[11-13]$ & $12[11-12]$ & 0.765 \\
\hline $\begin{array}{l}\text { Time to platelet } \\
\text { engraftment (days) }\end{array}$ & 12 [10-13] & $12[11-14]$ & 0.103 & 12 [11-13] & 12 [10-12] & 0.445 \\
\hline aGVHD & 17 (16.19\%) & 31 (35.23\%) & 0.002 & $12(42.86 \%)$ & 21 (53.85\%) & 0.375 \\
\hline cGVHD & $8(7.62 \%)$ & 9 (10.23\%) & 0.524 & $2(7.14 \%)$ & $8(20.51 \%)$ & 0.130 \\
\hline PRES & $3(2.86 \%)$ & $10(11.36 \%)$ & 0.022 & $3(10.71 \%)$ & $5(12.82 \%)$ & $>0.9999$ \\
\hline $\mathrm{HC}$ & 30 (28.57\%) & 16 (18.18\%) & 0.092 & $8(28.57 \%)$ & 12 (30.77\%) & 0.846 \\
\hline PTLD & $0(0.00 \%)$ & $1(1.14 \%)$ & 0.456 & $0(0.00 \%)$ & $1(2.56 \%)$ & $>0.9999$ \\
\hline CMV infection & $27(25.71 \%)$ & 23 (26.14\%) & 0.947 & $8(28.57 \%)$ & $13(33.33 \%)$ & 0.679 \\
\hline EBV infection & 15 (14.29\%) & 15 (17.05\%) & 0.598 & $6(21.43 \%)$ & $9(23.08 \%)$ & 0.873 \\
\hline VOD & $3(2.86 \%)$ & $1(1.14 \%)$ & 0.627 & $0(0.00 \%)$ & $2(5.13 \%)$ & 0.506 \\
\hline
\end{tabular}

GVHD, graft versus host disease; PRES, posterior reversible encephalopathy syndrome; HC, hemorrhagic cystitis; AlHA, autoimmune hemolytic anemia; BO, bronchiolitis obliterans; PTLD, posttransplant lymphoproliferative disorders; EBV, Epstein-Barr virus; VOD, vein occlusive disease.

Table 9 Multivariate analysis of factors associated with OS, TFS, GR and TRM

\begin{tabular}{llllr}
\hline & Factors & $\mathrm{P}$ & $\mathrm{HR}$ & \multicolumn{1}{c}{$95 \% \mathrm{Cl}$} \\
\hline OS & Patient risk classification & 0.009 & 3.56 & $1.38-9.22$ \\
& BO & 0.012 & 3.91 & 3.32 \\
TFS & CMV infection & 0.01 & 2.90 & $1.33-8.30$ \\
& Patient risk classification & 0.019 & 3.41 & $1.19-7.07$ \\
& BO & 0.020 & 2.36 & $1.21-9.59$ \\
GR & CMV infection & 0.045 & 17.93 & $1.02-5.45$ \\
& AlHA & 0.018 & 3.91 & $1.6-197.81$ \\
TRM & BO & 0.12 & 3.56 & $1.34-11.38$ \\
& Patient risk classification & 0.009 & 3.32 & $1.38-9.22$ \\
\hline
\end{tabular}

OS, overall survival; TFS, thalassemia-free survival; GR, graft rejection; TRM, transplantation-related mortality.

TRM (Table 9).

\section{Discussion}

A notable finding of this study was that allo-HSCT with the modified NF-08-TM conditioning regimen resulted in 6-year OS and TFS rates above $90 \%$ and a GR rate below $2 \%$. Importantly, there were no significant differences between the mismatched and fully-matched donor groups in time to neutrophil or platelet engraftment, incidence 
rates of complications (with the exception of aGVHD), OS, TFS, GR and TRM. Furthermore, multivariate analysis showed that HLA compatibility between patient and donor was not independently associated with OS, TFS, GR and TRM. Taken together, when modified NF-08TM conditioning regimen was applied in $\beta$-thalassemia major patients, mismatched-donor grafts could show similar survival outcomes and incidence rates of complications (except for aGVHD) as fully-matched donor counterparts after transplantation.

Although a matched sibling donor is generally considered to be ideal for allo-HSCT, the resource is limited. Alternative sources of cells for transplantation include matched unrelated donors, mismatched related donors, mismatched unrelated donors and cord blood (23).

Transplantation from a matched sibling donor is thought to reduce the incidence of GVHD. The use of bone marrow stem cells from fully matched siblings resulted in aGVHD and cGVHD incidence rates of $15.3 \%$ and $9.4 \%$, respectively (24). Qin et al. performed HSCT utilizing grafts from matched siblings' bone marrow, and demonstrated an aGVHD incidence of $12.8 \%$ and a cGVHD incidence of $14.9 \%$ (25). Importantly, patients treated by HSCT using matched sibling donors achieve the same quality of life as healthy people (26). In this study, the incidence of aGVHD for the fully-matched family donor group was higher than reported by others. However, none of the patients died of aGVHD, and all achieved satisfactory engraftment. We speculate that use of peripheral blood stem cells may result in a higher incidence of aGVHD than bone marrow application. Ghavamzadeh et al. concluded that peripheral blood stem cell transplantation is associated with higher incidence rates of aGVHD and cGVHD compared with bone marrow utilization, but blood cells are easier to harvest and associated with lower incidence of GR and shorter times to neutrophil and platelet engraftment, resulting in similar OS outcomes to bone marrow transplantation (27).

With the establishment of bone marrow banks worldwide, transplantation of grafts from unrelated donors has emerged as a viable alternative procedure. Shenoy et al. performed HSCT in patients utilizing matched unrelated marrow, and reported a 2 -year disease-free survival of $82 \%$ (28). A retrospective analysis reported higher incidence rates of aGVHD and cGVHD using matched unrelated donors compared with related donors, but with no significant differences in OS and TFS (8). This study found that use of the modified NF-08-TM conditioning regimen achieved OS and TFS exceeding $90 \%$, with no significant differences in outcomes between matched family and unrelated donors. Taken together, the current results and previous findings confirm that a matched unrelated donor graft is a safe and effective option.

The high incidence of GVHD has previously restricted the utilization of mismatched donors. In 2014, an international expert panel recommended that using an HLA-mismatched family donor should be considered an experimental approach; meanwhile, the unrelated donor must be selected using high-resolution molecular typing for both HLA class I and II loci, according to stringent criteria of compatibility with the recipient (29). Sun et al. performed HSCT in 48 patients using unrelated donors (HLA 9/10 in 16 patients and HLA 8/10 in 4 patients); notably, the incidence rates of aGVHD and cGVHD were low (30). A recent international survey reported worse outcome using HLA-mismatched related donors compared with HLA-mismatched unrelated donors (9). High-resolution mismatches at HLA-A, -B, -C, and -DRB1 adversely affect treatment outcome. With regards to HLA class II disparities, several studies have indicated that HLA-DQB1 disparities are not associated with mortality.

The present study found that the outcomes of transplantation using HLA-mismatched donors were similar to those using fully-matched donors while using the above conditioning regimen. Moreover, there were no significant differences in complication rates after transplantation between the two groups (except for aGVHD). Notably, the outcomes of transplantation using an HLA-mismatched family donor were similar to those of the HLA-mismatched unrelated donor group. Additionally, there were no significant differences in the incidence rates of aGVHD and cGVHD after transplantation between the two groups. We hypothesize that the satisfactory outcomes in this study were achieved because of the modified conditioning regimen adjusting the dose of anti-thymocyte globulin based on HLA compatibility between donor and recipient. Therefore, our findings provide strong evidence that while using this conditioning regimen, an HLA-mismatched donor is a suitable alternative to an HLA-identical donor.

Haploidentical donor transplantation brings challenges but also hope to patients. A study in Thailand described successful transplantation of peripheral blood stem cells from a haploidentical donor when a novel preconditioning regimen was used (31). Notably, continuous engraftment was achieved, with low incidence rates of severe complications and GVHD (31). A haploidentical transplantation study in China also obtained good outcomes: 3 -year OS and TFS 
reached $100 \%$, the incidence of grade III-IV aGVHD was low, and only 1 patient developed cGVHD (32). In this retrospective study, 22 patients received two or more HLA allele-mismatched grafts and four of them died at the last follow-up. Totally 15 patients received a graft from a parent, and three of them died at the last follow-up, indicating that parent donor-based transplantation is an alternative choice while using our modified NF-08-TM regimen. Since only a small number of patients received a parent graft in this study, additional studies are needed.

This was a retrospective analysis based on a registry, so the results may be prone to information or selection bias. In addition, the number of cases included was relatively small. A large-scale, multicenter, prospective clinical trial is needed to further evaluate the transplantation of fullymatched and mismatched donor grafts using the modified NF-08-TM regimen as well as new protocols.

In conclusion, when the modified NF-08-TM conditioning regimen and allo-HSCT were applied to treat $\beta$-thalassemia, survival outcomes and incidence rates of complications (except for aGVHD) were similar for mismatched and fully-matched donor grafts. Therefore, applying a mismatched graft is a safe and feasible choice when a suitable matched graft is unavailable for HSCT.

\section{Acknowledgments}

Funding: This work was supported by Guangzhou Women and Children's Medical Center.

\section{Footnote}

Reporting Checklist: The authors have completed the STROBE reporting checklist. Available at http://dx.doi. org/10.21037/tp-20-415

Data Sharing Statement: Available at http://dx.doi. org/10.21037/tp-20-415

Conflicts of Interest: All authors have completed the ICMJE uniform disclosure form (available at http://dx.doi. org/10.21037/tp-20-415). The authors have no conflicts of interest to declare.

Ethical Statement: The authors are accountable for all aspects of the work in ensuring that questions related to the accuracy or integrity of any part of the work are appropriately investigated and resolved. The study was conducted in accordance with the Declaration of Helsinki (as revised in 2013). It was approved by the ethics committee of Guangzhou Women and Children's Medical Center (No. 2013092569), and individual consent for this retrospective analysis was waived.

Open Access Statement: This is an Open Access article distributed in accordance with the Creative Commons Attribution-NonCommercial-NoDerivs 4.0 International License (CC BY-NC-ND 4.0), which permits the noncommercial replication and distribution of the article with the strict proviso that no changes or edits are made and the original work is properly cited (including links to both the formal publication through the relevant DOI and the license). See: https://creativecommons.org/licenses/by-nc-nd/4.0/.

\section{References}

1. Origa R. beta-Thalassemia. Genet Med 2017;19:609-19.

2. de Dreuzy E, Bhukhai K, Leboulch P, et al. Current and future alternative therapies for beta-thalassemia major. Biomed J 2016;39:24-38.

3. Porter J. Beyond transfusion therapy: new therapies in thalassemia including drugs, alternate donor transplant, and gene therapy. Hematology Am Soc Hematol Educ Program 2018;2018:361-70.

4. Choudhary D, Doval D, Sharma SK, et al. Allogenic Hematopoietic Cell Transplantation in Thalassemia Major: A Single-center Retrospective Analysis From India. J Pediatr Hematol Oncol 2019;41:e296-e301.

5. Huang K, Zhou DH, Li Y, et al. Modified conditioning regimen improves outcomes of unrelated donor peripheral blood stem cell transplantation for beta-thalassaemia major patients. Pediatr Blood Cancer 2018;65:e27026.

6. Baronciani D, Angelucci E, Potschger U, et al. Hemopoietic stem cell transplantation in thalassemia: a report from the European Society for Blood and Bone Marrow Transplantation Hemoglobinopathy Registry, 2000-2010. Bone Marrow Transplant 2016;51:536-41.

7. He Y, Jiang $\mathrm{H}, \mathrm{Li} \mathrm{C}$, et al. Long-term results of the NF08-TM protocol in stem cell transplant for patients with thalassemia major: A multi-center large-sample study. Am J Hematol 2020;95:E297-E299.

8. John MJ, Mathew A, Philip CC, et al. Unrelated and related donor transplantation for beta-thalassemia major: A single-center experience from India. Pediatr Transplant 2018;22:e13209.

9. Li C, Mathews V, Kim S, et al. Related and unrelated 
donor transplantation for beta-thalassemia major: results of an international survey. Blood Adv 2019;3:2562-70.

10. Oved JH, Wang Y, Barrett DM, et al. CD3(+)/ CD19(+) Depleted Matched and Mismatched Unrelated Donor Hematopoietic Stem Cell Transplant with Targeted T Cell Addback Is Associated with Excellent Outcomes in Pediatric Patients with Nonmalignant Hematologic Disorders. Biol Blood Marrow Transplant 2019;25:549-55.

11. Woolfrey A, Klein JP, Haagenson M, et al. HLA-C antigen mismatch is associated with worse outcome in unrelated donor peripheral blood stem cell transplantation. Biol Blood Marrow Transplant 2011;17:885-92.

12. Li C, Wu X, Feng X, et al. A novel conditioning regimen improves outcomes in beta-thalassemia major patients using unrelated donor peripheral blood stem cell transplantation. Blood 2012;120:3875-81.

13. Pistoia L, Meloni A, Ricchi P, et al. Genotypic groups as risk factors for cardiac magnetic resonance abnormalities and complications in thalassemia major: a large, multicentre study. Blood Transfus 2021;19:168-76.

14. Mediwake H, Curley C, Butler J, et al. Mismatched unrelated donor allogeneic stem cell transplant for high risk haematological malignancy: A single centre experience. Blood Cancer J 2017;7:655.

15. Ansari M, Huezo-Diaz P, Rezgui MA, et al. Influence of glutathione S-transferase gene polymorphisms on busulfan pharmacokinetics and outcome of hematopoietic stemcell transplantation in thalassemia pediatric patients. Bone Marrow Transplant 2016;51:377-83.

16. Gaziev J, Nguyen L, Puozzo C, et al. Novel pharmacokinetic behavior of intravenous busulfan in children with thalassemia undergoing hematopoietic stem cell transplantation: a prospective evaluation of pharmacokinetic and pharmacodynamic profile with therapeutic drug monitoring. Blood 2010;115:4597-604.

17. Watanabe E, Nishikawa T, Ikawa K, et al. Trough level monitoring of intravenous busulfan to estimate the area under the plasma drug concentration-time curve in pediatric hematopoietic stem cell transplant recipients. Int J Hematol 2015;102:611-6.

18. Admiraal R, Boelens JJ. Anti-thymocyte globulin for GVHD: one dose does not fit all. Lancet Haematol 2020;7:e505.

19. Kröger N, Solano C, Bonifazi F. Antilymphocyte Globulin for Chronic Graft-versus-Host Disease. N Engl J Med 2016;374:1894-5.

20. Philippe M, Goutelle S, Guitton J, et al. Should busulfan therapeutic range be narrowed in pediatrics? Experience from a large cohort of hematopoietic stem cell transplant children. Bone Marrow Transplant 2016;51:72-8.

21. Przepiorka D, Weisdorf D, Martin P, et al. 1994 Consensus Conference on Acute GVHD Grading. Bone Marrow Transplant 1995;15:825-8.

22. Shulman HM, Sullivan KM, Weiden PL, et al. Chronic graft-versus-host syndrome in man. A long-term clinicopathologic study of 20 Seattle patients. Am J Med 1980;69:204-17.

23. Locatelli F, Merli P, Strocchio L. Transplantation for thalassemia major: alternative donors. Curr Opin Hematol 2016;23:515-23.

24. Li Q, Luo J, Zhang Z, et al. G-CSF-Mobilized Blood and Bone Marrow Grafts as the Source of Stem Cells for HLA-Identical Sibling Transplantation in Patients with Thalassemia Major. Biol Blood Marrow Transplant 2019;25:2040-4.

25. Qin F, Shi L, Li Q, et al. Immune recovery after in vivo T-cell depletion myeloablative conditioning hematopoietic stem cell transplantation in severe beta-thalassemia children. Eur J Haematol 2019;103:342-50.

26. Caocci G, Vacca A, Piras E, et al. Return to normal life after hematopoietic stem cell transplantation for thalassemia: a study of patients transplanted from matched sibling donors. Bone Marrow Transplant 2016;51:1640-1.

27. Ghavamzadeh A, Kasaeian A, Rostami T, et al. Comparable Outcomes of Allogeneic Peripheral Blood versus Bone Marrow Hematopoietic Stem Cell Transplantation in Major Thalassemia: A Multivariate Long-Term Cohort Analysis. Biol Blood Marrow Transplant 2019;25:307-12.

28. Shenoy S, Thompson AA. Unrelated donor stem cell transplantation for transfusion-dependent thalassemia. Ann N Y Acad Sci 2016;1368:122-6.

29. Angelucci E, Matthes-Martin S, Baronciani D, et al. Hematopoietic stem cell transplantation in thalassemia major and sickle cell disease: indications and management recommendations from an international expert panel. Haematologica 2014;99:811-20.

30. Sun L, Wang N, Chen Y, et al. Unrelated Donor Peripheral Blood Stem Cell Transplantation for Patients with beta-Thalassemia Major Based on a Novel Conditioning Regimen. Biol Blood Marrow Transplant 2019;25:1592-6.

31. Anurathapan U, Hongeng S, Pakakasama S, et al. Hematopoietic stem cell transplantation for homozygous 
beta-thalassemia and beta-thalassemia/hemoglobin E patients from haploidentical donors. Bone Marrow Transplant 2016;51:813-8.

32. Sun Q, Wu B, Lan H, et al. Haploidentical haematopoietic

Cite this article as: Huang C, Qu Y, Liu S, Nie S, Jiang H. Hematopoietic stem cell transplantation for thalassemia major using HLA fully-matched and mismatched donor grafts. Transl Pediatr 2021;10(6):1552-1565. doi: 10.21037/tp-20-415 stem cell transplantation for thalassaemia major based on an FBCA conditioning regimen. Br J Haematol 2018;182:554-8. 